\title{
MicroRNA-615-5p targets insulin-like growth factor 2 and exerts tumor-suppressing functions in human esophageal squamous cell carcinoma
}

\author{
BINGYIN YANG, RUI XIE, SHANG-NONG WU, CHENG-CHENG GAO, \\ XIAO-ZHONG YANG and JING-FANG ZHOU \\ Department of Gastroenterology, Huai'an First People's Hospital, \\ Nanjing Medical University, Huai'an, Jiangsu 223300, P.R. China
}

Received November 1, 2016; Accepted April 18, 2017

DOI: 10.3892/or.2017.6079

\begin{abstract}
To investigate the expression pattern, clinical significance and functional roles of microRNA (miR)-615-5p in human esophageal squamous cell carcinoma (ESCC), , quantitative real-time PCR was performed to detect expression levels of miR-615-5p in ESCC tissues and cell lines. Associations between miR-615-5p expression and various clinicopathological features of ESCC patients were also statistically evaluated. The candidate targets of miR-615-5p were identified by integrating bioinformatics miRNA target prediction, western blot analysis and luciferase reporter assay. Moreover, the functions of miR-615-5p in ESCC cell migration and invasion were determined using the transfection of miRNA mimics, or co-transfected with miRNA mimics and the expression vector of its target gene. As a result, miR-615-5p expression in ESCC tissues and cells were markedly lower than those in non-cancerous esophageal mucosa and human normal esophageal cells, respectively (both $\mathrm{P}<0.001$ ). miR-615-5p downregulation was significantly associated with advanced tumor-node-metastasis stage, positive lymph node metastasis and moderate-poor differentiation. Functionally, the re-expression of miR-615-5p suppressed the invasion and migration of ESCC cells in vitro. Interestingly, insulin-like growth factor 2 (IGF2) was identified as a direct target gene of miR-615-5p, and the inhibitory effects of miR-615-5p in ESCC cell motility were reversed by the restoration of IGF2 expression. In conclusion, miR-615-5p downregulation may be an underlying molecular mechanism of development and progression of ESCC, and may function as a potential therapeutic target of this malignancy. Also, we illustrate that the
\end{abstract}

Correspondence to: Professor Jing-Fang Zhou, Department of Gastroenterology, Huai'an First People's Hospital, Nanjing Medical University, Huai'an, Jiangsu 223300, P.R. China

E-mail: jingfangzhou116@163.com

Key words: esophageal squamous cell carcinoma, microRNA-615-5p, insulin-like growth factor 2, cell motility, carcinogenesis
miR-615-5p/IGF2 axis may bring important contributions to cell motility of human ESCC.

\section{Introduction}

Human esophageal cancer, as one of the most common malignancies, it ranks eighth in incidence and sixth in cancer-related death worldwide (1). It contains two principle histopathologic subtypes, including esophageal adenocarcinoma and esophageal squamous cell carcinoma (ESCC) (2), the latter predominantly occurs in eastern and southern Africa, and eastern Asia (3). Despite great advances in early diagnosis and treatment strategies of human ESCC during the past twenty years, the clinical outcome of patients with this malignancy remains very poor, with estimated 5-year survival rate at $<25 \%$ (4). Emerging evidence indicates that esophagealcarcinogenesis is governed by various molecular processes such as dysregulation of oncogenes or tumor suppressor genes which were observed frequently in ESCC tissues leading to their aberrant expression and increased cellular proliferation, cell cycle progression and cell motility $(5,6)$. Therefore, it is crucial to identify novel markers for early detection and efficient treatment, and to understand the underlying mechanisms of carcinogenesis and cancer progression of human ESCC.

MicroRNAs (miRNAs), a group of newly discovered endogenous, non-coding, single stranded and short RNAs with 18-29 nucleotides in length, has been proved to play roles in various cellular processes, including cell development, differentiation, proliferation, metabolism and malignant transformation (7). miRNAs can negatively regulate expression levels of the corresponding target genes at a post-transcriptional level by promoting mRNA degradation or suppressing translation (8). Accumulating studies have identified a number of miRNAs which may be involved into the initiation and progression of human cancers (9). Especially, Liu et al (10) proved that miR-373 could promote migration and invasion in human ESCC by inhibiting TIMP3 expression. Results of a previous study reported by Nie et al (11) showed that miR-34a was associated with ESCC migration and inhibited the migration and invasion of ESCC cells by targeting Yin Yang-1; Mao et al (12) indicated that miR-1290 might function as an 
oncogene in the progression of ESCC by targeting nuclear factor I/X. These findings imply that the dysregulation of miRNAs may contribute to several malignant processes including cancer cell cycle, apoptosis, invasion, migration and metastasis by affecting the corresponding transcripts (13). miR-615-5p, located in $\mathrm{CpG}$ islands of the HOX gene cluster on chromosome 12q13.13, has been determined to be frequently downregulated and functions as a tumor suppressor in various human cancers via specially binding to the complimentary recognition sequences in the 3'-untranslated region (3'UTR) of the corresponding target mRNAs (14-16). However, little is known regarding its involvement in human ESCC.

To address this problem, we performed quantitative realtime PCR to detect expression levels of miR-615-5p in both ESCC tissues and cell lines. Then, associations between miR-615-5p expression and various clinicopathological features of ESCC patients were statistically evaluated. Then the candidate targets of miR-615-5p were identified by integrating bioinformatics miRNA target prediction, western blot analysis and luciferase reporter assay. In addition, the functions of miR-615-5p in ESCC cell migration and invasion were determined using the transfection of miRNA mimics, or co-transfected with miRNA mimics and the expression vector of its target gene.

\section{Materials and methods}

Ethics approval. All procedures performed in studies involving human participants were in accordance with the ethical standards of the Institutional and/or National Research Committee and with the 1964 Helsinki declaration and its later amendments or comparable ethical standards.

Ethic statement. The present study was approved by the Ethic Committee of Huai'an First People's Hospital. Prior informed consent was signed by all the patients enrolled in this study based on the guidelines of Huai'an First People's Hospital. All clinical tissue specimens were handled and made anonymous according to the ethical and legal standards.

Patients and tissue samples. Sixty primary ESCC and matched adjacent non-cancerous esophageal mucosa tissues were obtained from 60 ESCC patients in the Department of Gastroenterology, Huai'an First People's Hospital between January 2012 and December 2015. All ESCC patients underwent esophagectomy, and none of them received preoperative radiotherapy or chemotherapy. The diagnosis of ESCC patients was confirmed by clinical examination and histopathological analysis of the tissue specimens. The clinicopathological characteristics of all 60 ESCC patients, including age, sex, tumor location, lymph node metastasis, Tumor-node-metastasis (TNM) stage and pathological grade are summarized in Table I. All the samples were collected and immediately snap-frozen in liquid nitrogen and stored at $-80^{\circ} \mathrm{C}$ until further use.

Cell lines and culture. Human normal esophageal cell line (HEEC) and two human ESCC cell lines (ECA109 and KYSE410) were purchased from the Shanghai Institute of Cell Biology, Chinese Academy of Sciences (Shanghai, China). All cell lines were cultured in Dulbecco's modified Eagle's medium (DMEM; Gibco; Thermo Fisher Scientific, Inc., Waltham, MA, USA) supplemented with $10 \%$ fetal bovine serum. Cells were cultured at $37^{\circ} \mathrm{C}$ with $5 \% \mathrm{CO}_{2}$ for further use.

Cell transfection. miR-615-5p and negative control mimics (miR-615-5p/NC_mimics) were purchased from GeneCopoeia (Guangzhou, China). The IGF2 expression plasmid was constructed using a PCR-generated fragment from the mRNA and the Lv-CMV-GFP vector (en-IGF-2), and the negative control (en-NC) had no insert. Two human ESCC cell lines were transfected with miR-615-5p/NC_mimics or en-IFG-2/en-NC by Lipofectamine 2000 (Invitrogen, Carlsbad, CA, USA) according to the manufacturer's instructions. Forty-eight hours after the transfection, ESCC cells were harvested for western blot or real-time quantitative PCR analyses.

Western blot analysis. Proteins were extracted from fresh clinical tissue specimens and cells using cell lysis buffer (50 mM Tris-HCl, pH 8.0, 2 mM EDTA, 1 mM DTT, $10 \mathrm{mM}$ $\mathrm{NaCl}, 5 \mathrm{mg} / \mathrm{ml}$ leupeptin, $1 \% \mathrm{NP}-40,2 \mathrm{mg} / \mathrm{ml}$ pepstatin, $2 \mathrm{mg} / \mathrm{ml}$ aprotinin, $0.1 \%$ SDS and $1 \mathrm{mM}$ phenylmethylsulfonyl fluoride). Equal amounts of protein (50 $\mu \mathrm{g})$ were separated by $10 \%$ SDS PAGE and then transferred onto polyvinylidene difluoride membranes (Qiagen China Co., Ltd.). Then, the membranes were incubated with the primary antibodies: antiIGF2 (dilution 1:500, Abcam Inc., MA, USA) and anti-GAPDH (dilution 1:500, Abcam Inc.), after blocking with $8 \%$ milk in phosphate-buffered saline (PBS; pH 7.5). After that, the membranes were incubated with the appropriate horseradish peroxidase-conjugated secondary antibodies (dilution 1:1,000, Abcam Inc.) after the incubation at $4^{\circ} \mathrm{C}$ overnight. Finally, protein was visualized using enhanced chemiluminescence reagent (Santa Cruz Biotechnology, Santa Cruz, CA, USA). The expression level of IGF2 protein was normalized to that of GAPDH protein. Each sample was examined in triplicate.

$R N A$ extraction and real-time quantitative RT-PCR. Total RNA in fresh clinical tissue specimens and cells were extracted using the RNeasy RNA Mini kit (Qiagen GmbH, Hilden, Germany) according to the manufacturer's instructions. First strand cDNA was synthesized using SuperScript reverse transcriptase (Clontech Laboratories, Inc., Mountainview, CA, USA) according to the manufacturer's instructions. Following the cDNA synthesis, the real-time PCR was performed using a Fast Start Master SYBR Green kit (Roche Molecular Systems, Indianapolis, IN, USA) on a LightCycler (Roche Molecular Systems) according to the manufacturer's instructions. The sequence-specific primer pairs were used for quantitative PCR and are as follows: miR-615-5p forward, 5'-TCC GAT TCT CCC TCT GGG TC-3'; reverse, 5'-GTG CAG GGT CCG AGG T-3'. U6 forward, 5'-CTC GCT TCG GCA GCA CA-3'; reverse, 5'-AAC GCT TCA CGA ATT TGC GT-3'. IGF2 forward, 5'-CCT TGG ACT TTG AGT CAA ATT-3'; reverse, 5'-GGT CGT GCC AAT TAC ATT TCA-3'. GAPDH forward, 5'-GCT GAG TAT GTC GTG GAG TC-3'; reverse, 5'-AGT TGG TGG TGC AGG ATG C-3'. Relative expression levels of miRNA and mRNA expression were calculated with the $2^{-\Delta \Delta C t}$ method in fresh tissues and cells. Each sample was examined in triplicate. 
Cell invasion and migration assays. Cell migration and invasion abilities of human ESCC cell lines with the transfection of miR-615-5p/NC-mimics or with the co-transfection of miR-615-5p/en-IGF2 were evaluated using a Millicell Transwell chamber (Millipore, Billerica, MA, USA), with or without Matrigel (BD Biosciences, Franklin Lakes, NJ, USA). For the invasion and migration assays, $48 \mathrm{~h}$ following the transfection, Transwell chambers were placed into 24-well plates which were respectively precoated with or without a 5-ml mixture of BD Matrigel and DMEM (1:1, v/v). Following incubation at $37^{\circ} \mathrm{C}$ in a humidified incubator with $5 \% \mathrm{CO}_{2}$ for $40 \mathrm{~min}$, for cell migration and invasion assays, $1 \times 10^{5}$ tumor cells in $0.1 \mu \mathrm{l}$ of media without FBS were plated in the upper chamber. In the lower chamber, $0.6 \mu \mathrm{l}$ of the medium with $10 \%$ FBS was added. Forty-eight hours after the incubation, cells on the upper surface of the Millicell chambers, noninvasive or migrated cells, were removed with a cotton swab. Tumor cells on the bottom surface of the membrane were fixed with $4 \%$ paraformaldehyde at room temperature for $30 \mathrm{~min}$ and stained with $0.1 \%$ crystal violet for $15 \mathrm{~min}$. The number of migrated or invasive cells was counted in five randomly selected fields under an inverted microscope (Olympus CKX41; Olympus Corporation, Tokyo, Japan). Each sample was examined in triplicate.

miRNA target prediction. miRTarBase (Release 6.1; http:// mirtarbase.mbc.nctu.edu.tw/) was used to collect the validated target genes of miR-615-5p. miRTarBase has accumulated more than 360,000 MTIs validated experimentally by reporter assay, western blot analysis, qPCR, microarray and next-generation sequencing experiments (17). This study only collected the MTIs which are validated experimentally by reporter assay, western blot and qPCR analyses.

Luciferase reporter assay. Two human ESCC cell lines ECA109 and KYSE410 $\left(4.0 \times 10^{4}\right)$ were cultured in 24-well plates. The fragments of ligating oligonucleotides with the wildtype (WT) or mutant-type (MUT) putative binding site of the human IGF2 3'-UTR were cloned between the Fse1 and Xba1 sites of pGL3-control (Promega, Madison, WI, USA). Then, the cells were co-transfected with miR-615-5p/NC-mimics and pGL3-IGF2-3'-UTR-WT/MUT using Lipofectamine 2000 (Invitrogen). Forty-eight hours following the transfection, ECA109 and KYSE410 cells were collected and detected with the Dual-Luciferase Reporter assay system (Promega). Luciferase activity was measured by a Lumat LB 9507 apparatus (Berthold Technologies $\mathrm{GmbH} \& \mathrm{Co}$. KG, Bad Wildbad, Germany). Each sample was examined in triplicate.

Statistical analysis. All data were described as mean \pm standard deviation (SD) and statistically analyzed using SPSS software (version 11.0, SPSS, Inc., Chicago, IL, USA). The differences between groups were analyzed using Student's t-test. Spearman's correlation analysis was performed to assess the correlation between miR-615-5p expression and IGF2 mRNA expression in ESCC tissues. The associations between miR-615-5p expression and various clinicopathological characteristics of ESCC patients were performed using the $\chi^{2}$ test for categorical variables. Difference between groups was considered to be statistically significant at $\mathrm{P}<0.05$.
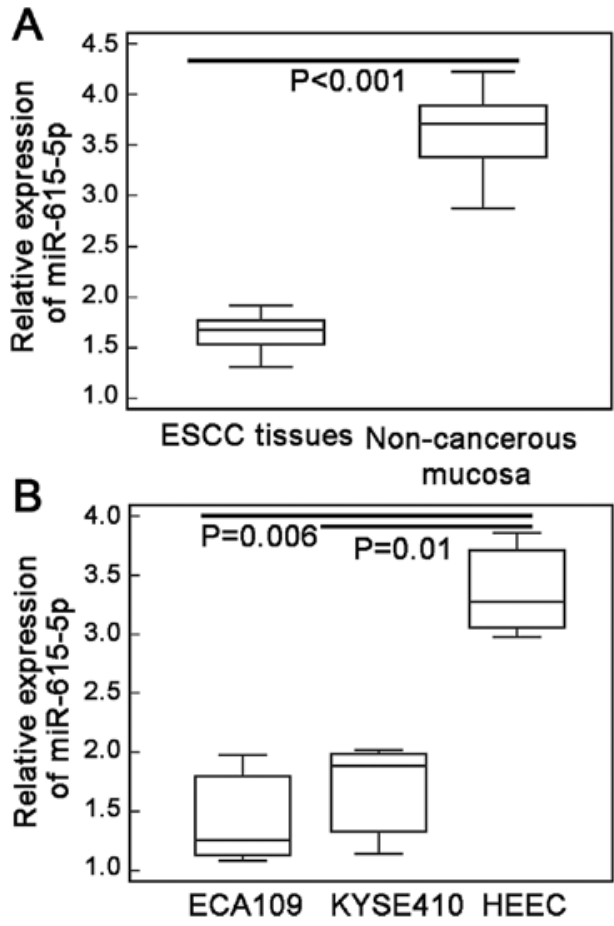

Figure 1. Reduced expression of microRNA (miR)-615-5p in ESCC tissues and cells. Quantitative real-time PCR was performed to detect expression levels of miR-615-5p in ESCC tissues and cell lines. (A) miR-615-5p expression in ESCC tissues was markedly lower than those in adjacent non-cancerous esophageal mucosa tissues (ESCC vs. non-cancerous tissues: $1.70 \pm 0.30$ vs. $3.62 \pm 0.39$, $\mathrm{P}<0.001$ ). (B) miR-615-5p expression in two human ESCC cell lines ECA109 and KYSE410 were markedly lower than those in human normal esophageal cell line (HEEC) (ECA109 vs. HEEC cells: $1.46 \pm 0.42$ vs. 3.38 $\pm 0.45, \mathrm{P}=0.006$; KYSE410 vs. HEEC cells: $1.69 \pm 0.46$ vs. $3.38 \pm 0.45, \mathrm{P}=0.01)$.

\section{Results}

Reduced expression of miR-615-5p in both ESCC tissues and cells. miR-615-5p expression in ESCC tissues, and two human ESCC cell lines ECA109 and KYSE410 were markedly lower than those in adjacent non-cancerous esophageal mucosa and human normal esophageal cell line (HEEC) (ESCC vs. noncancerous tissues: $1.70 \pm 0.30$ vs. $3.62 \pm 0.39, \mathrm{P}<0.001$, Fig. $1 \mathrm{~A}$; ECA109 vs. HEEC cells: $1.46 \pm 0.42$ vs. $3.38 \pm 0.45, \mathrm{P}=0.006$; KYSE410 vs. HEEC cells: $1.69 \pm 0.46$ vs. $3.38 \pm 0.45, \mathrm{P}=0.01$; Fig. 1B).

Reduced expression of miR-615-5p associates with aggressive tumor progression of ESCC patients. To evaluate the associations between miR-615-5p expression and various clinicopathological features of ESCC patients, we divided the 60 patients into low miR-615-5p expression $(n=31)$ and high miR-615-5p expression $(n=29)$ groups using the median value of miR-615-5p (1.68) expression levels in ESCC tissues as a cutoff point. As shown in Table I, ESCC patients with positive lymph node metastasis $(\mathrm{P}=0.01$, Table I), moderate-poor differentiation $(\mathrm{P}=0.03$, Table $\mathrm{I})$ and advanced TNM stage $(\mathrm{P}<0.001$, Table I) had lower expression of miR-615-5p than those with negative lymph node metastasis, well differentiation and early TNM stage. However, no significant association between miR-615-5p expression and the patient age or sex, and tumor location was found (all $\mathrm{P}>0.05$, Table I). 


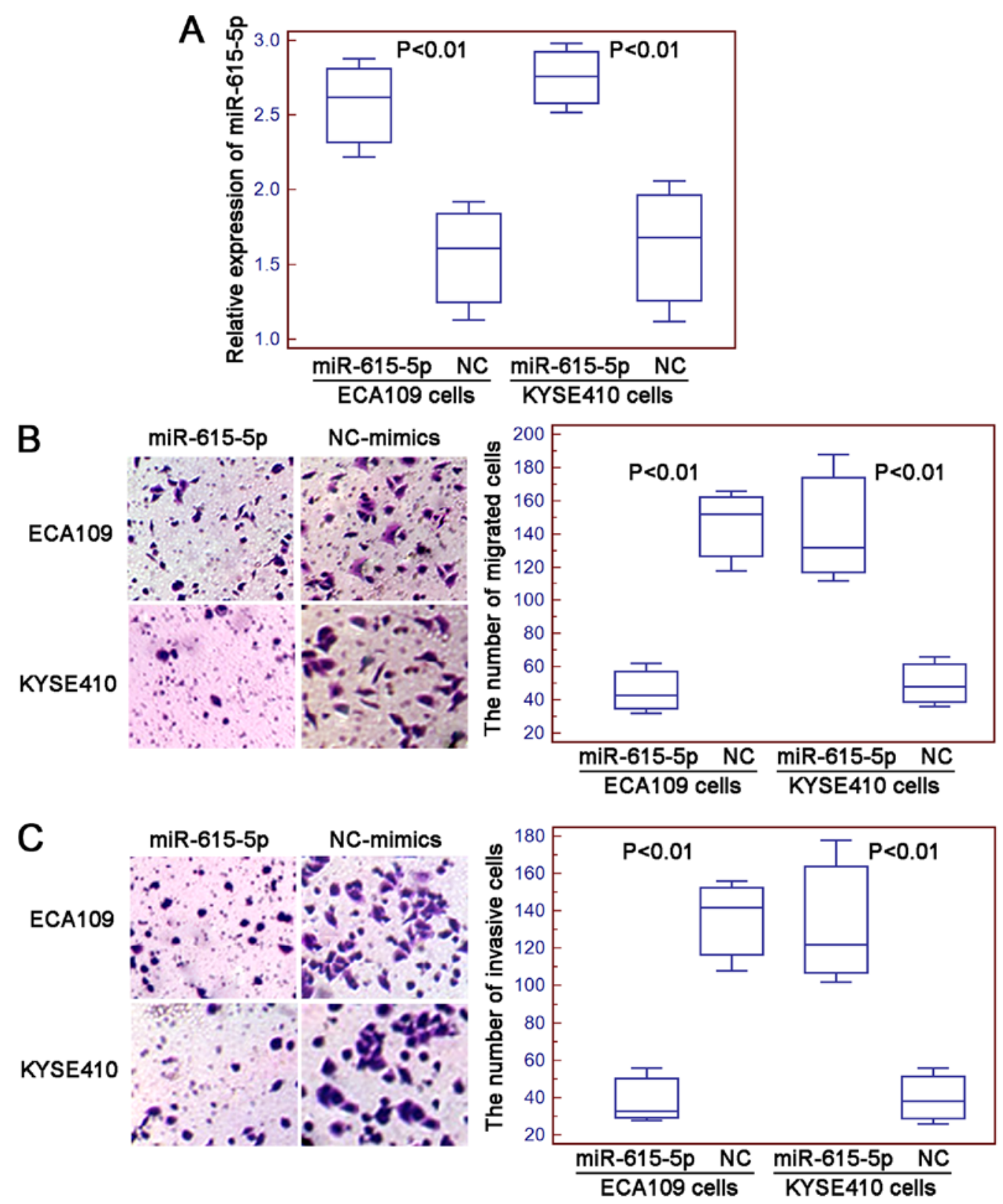

Figure 2. miR-615-5p suppresses cell motility of human ESCC cells in vitro. (A) The transfection efficiency was evaluated using quantitative real-time PCR and the results indicated that the expression levels of miR-615-5p in ECA109 and KYSE410 cells were both significantly increased by the transfection with miR-615-5p mimics compared with negative control mimics (both $\mathrm{P}<0.01$ ). (B) Transwell assays were performed to detect the functions of miR-615-5p in ESCC cell migration and invasion. The number of migrated ESCC cells was markedly decreased in cells transfected with miR-615-5p mimics compared to the cells transfected with negative control mimics (for ECA109 and KYSE410 cells: both P<0.01). (C) The number of invasive ESCC cells was markedly decreased in cells transfected with miR-615-5p mimics compared to the cells transfected with negative control mimics (for ECA109 and KYSE410 cells: both P<0.01).

miR-615-5p suppresses cell motility of human ESCC cells in vitro. The migration and invasion of ECA109 and KYSE410 cells after the transfection with miR-615-5p mimics or negative control mimics were determined using the Transwell assays. The transfection efficiency was evaluated using quantitative real-time PCR and the results indicated that the expression levels of miR-615-5p in ECA109 and KYSE410 cells were both significantly increased by the transfection with miR-615-5p mimics compared with negative control mimics (both $\mathrm{P}<0.01$, Fig. $2 \mathrm{~A}$ ). Then, the numbers of migrated and invasive ESCC cells were markedly decreased in cells transfected with miR-615-5p mimics compared to the cells transfected with negative control mimics (for ECA109 and KYSE410 cells: both $\mathrm{P}<0.01$, Fig. $2 \mathrm{~B}$ and C).
$I G F 2$ is a direct target gene of miR-615-5p. To investigate the underlying mechanism of the inhibitory effect of miR-615-5p in ESCC cell motility, we collected its candidate targets from miRTarBase and found that the interaction between miR-615-5p and IGF2 were validated experimentally by reporter assay in hepatocellular carcinoma cells (18).

Next, we performed luciferase report assay based on two human ESCC cell lines to confirm the binding efficiency between miR-615-5p and IGF2 in ECA109 and KYSE410 cells. As a result, ESCC cells co-transfected with miR-615-5pmimics and pGL3-IGF2-3'-UTR-WT both showed significantly lower luciferase reporter activity than those co-transfected with NC-mimics and pGL3-IGF2-3'-UTR-WT plasmid. However, there was no difference with statistical 
A
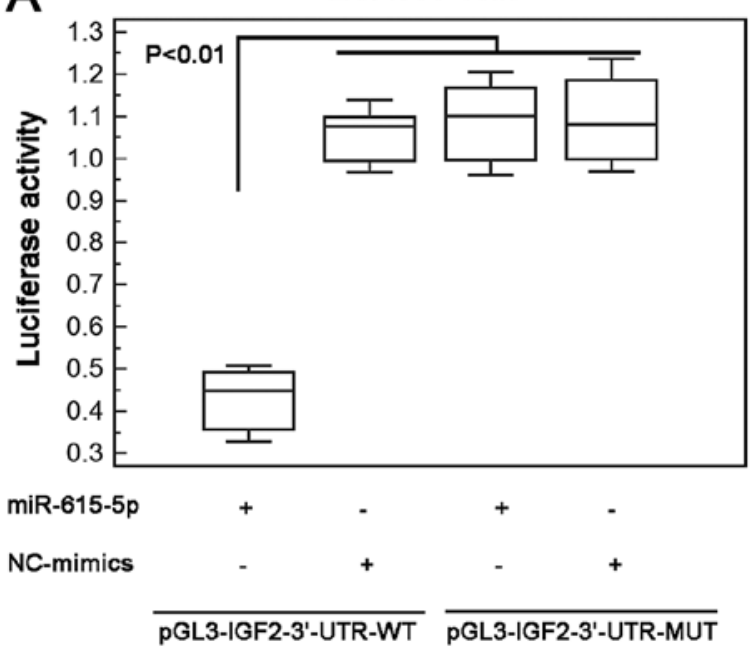

B
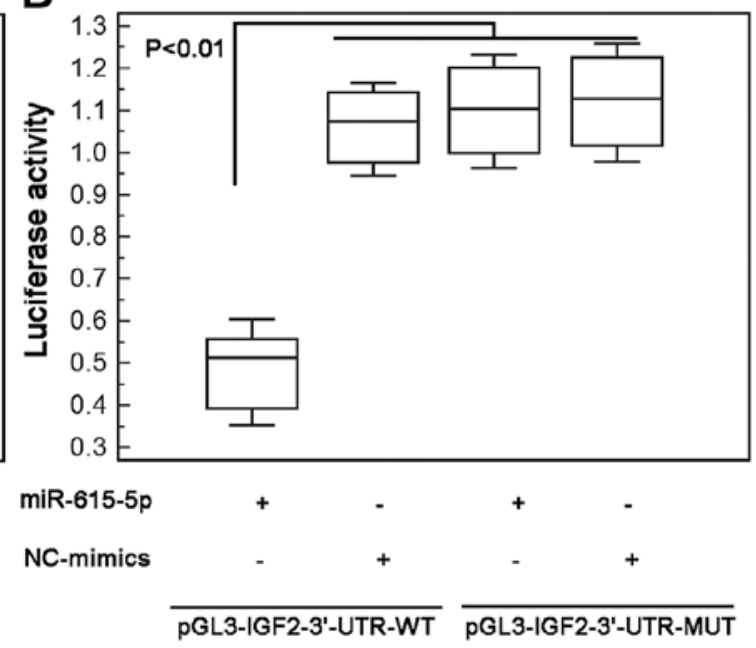

D
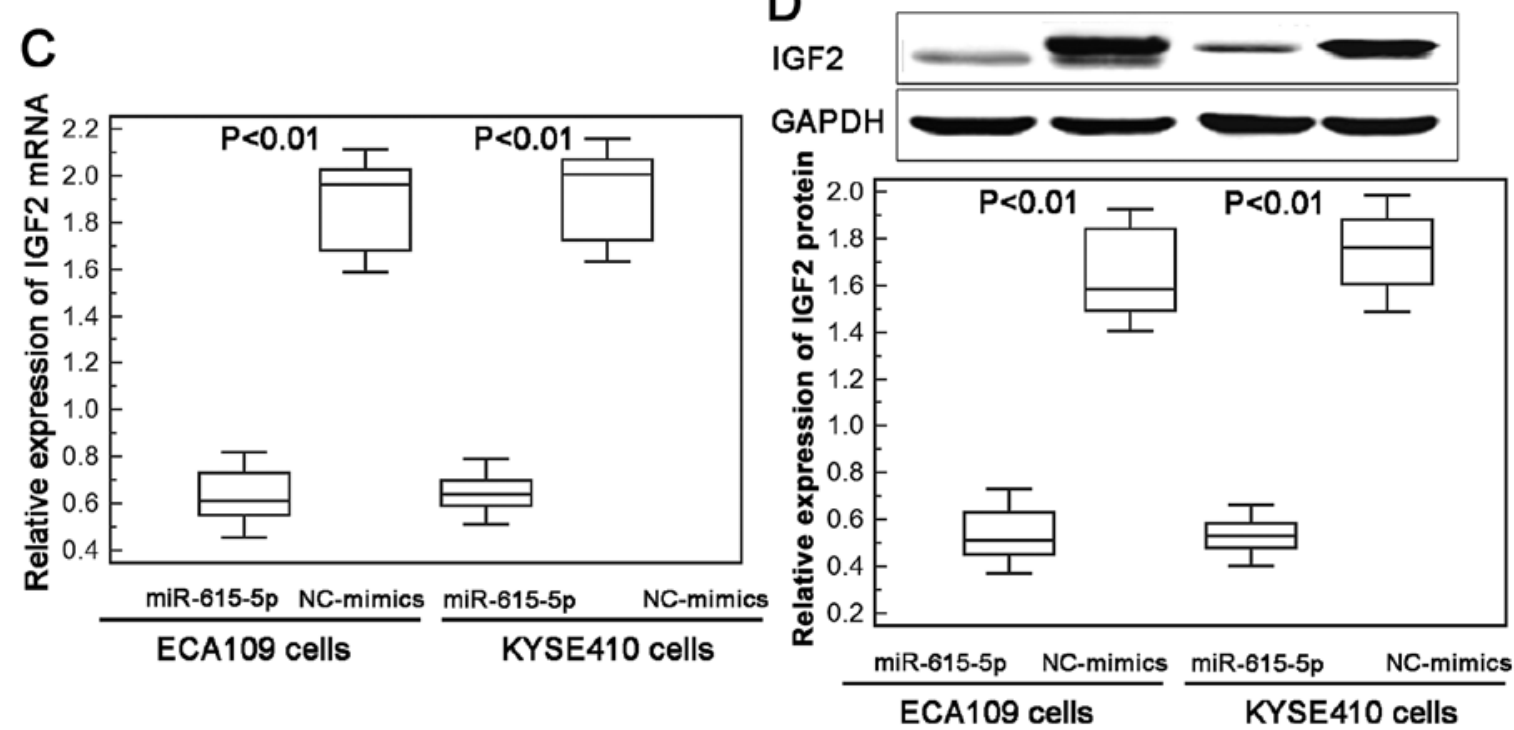

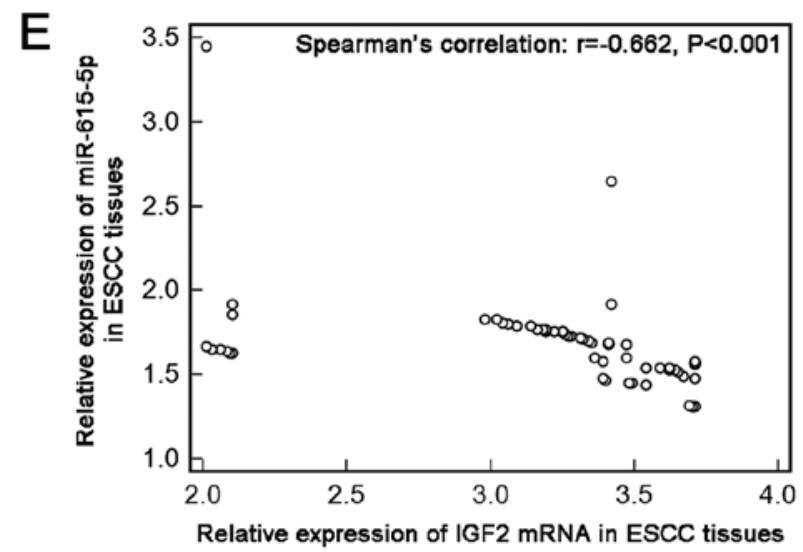

Figure 3. IGF2 is a direct target gene of miR-615-5p. (A and B) The luciferase reporter activities in ECA109 and KYSE410 cells with the co-transfection of miR-615-5p-mimics and pGL3-IGF2-3'-UTR-WT were both significantly lower than those with the co-transfection of NC-mimics and pGL3-IGF23'-UTR-WT. (C and D) Re-expression of miR-615-5p efficiently suppressed the endogenous expression of IGF2 at both mRNA and protein level in ECA109 and KYSE410 cells. (E) Spearman's correlation analysis showed a negative correlation between miR-615-5p and IGF2 mRNA expression in ESCC tissues (Spearman's correlation: $\mathrm{r}=-0.662, \mathrm{P}<0.001)$.

significance in the reporter activity between ECA109 and KYSE410 cells co-transfected with miR-615-5p-mimics and pGL3-IGF2-3'-UTR-MUT plasmid and cells co-transfected with NC-mimics and pGL3-IGF2-3'-UTR-MUT plasmid (Fig. 3A and B).
Moreover, the re-expression of miR-615-5p efficiently suppressed the endogenous expression of IGF2 at both mRNA and protein levels in ECA109 and KYSE410 cells (Fig. 3C and D). Further Spearman's correlation analysis displayed a negative correlation between miR-615-5p and IGF2 
Table I. Associations between miR-615-5p expression and various clinicopathological characteristics of 60 patients with ESCCs.

\begin{tabular}{lcc}
\hline Clinical variables & No. of patients $(\%)$ & Low miR-615-5p expression \\
\hline Age (year) & & \\
$\leq 60$ & $25(41.67)$ & $13(52.00)$ \\
$>60$ & $35(58.33)$ & $18(51.43)$ \\
Sex & & $21(52.50)$ \\
Male & $40(66.67)$ & $10(50.00)$ \\
Female & $20(33.33)$ & \\
Tumor location & & $22(53.66)$ \\
Upper 1/3-middle $1 / 3$ & $41(68.33)$ & $9(47.37)$ \\
Lower $1 / 3$ & $19(31.67)$ & $6(25.00)$ \\
Lymph node metastasis & & $25(69.44)$ \\
Negative & $24(40.00)$ & $2(10.00)$ \\
Positive & $36(60.00)$ & $29(72.50)$ \\
TNM stage & & NSS \\
Absent & $20(33.33)$ & $6(33.33)$ \\
Present & $40(66.67)$ & $25(59.52)$ \\
Histological differentiation & $18(33.33)$ & 0.01 \\
Well & $42(66.67)$ & $<0.001$ \\
Moderate-poor & & \\
\hline
\end{tabular}

A
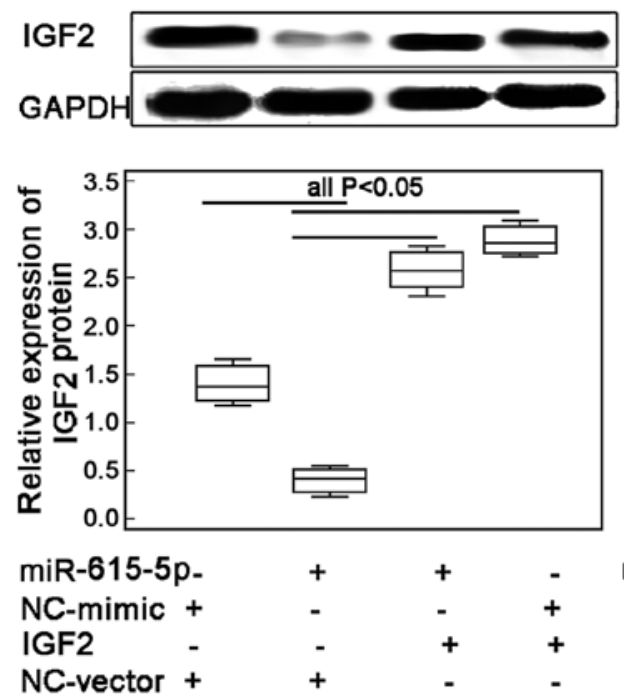

B
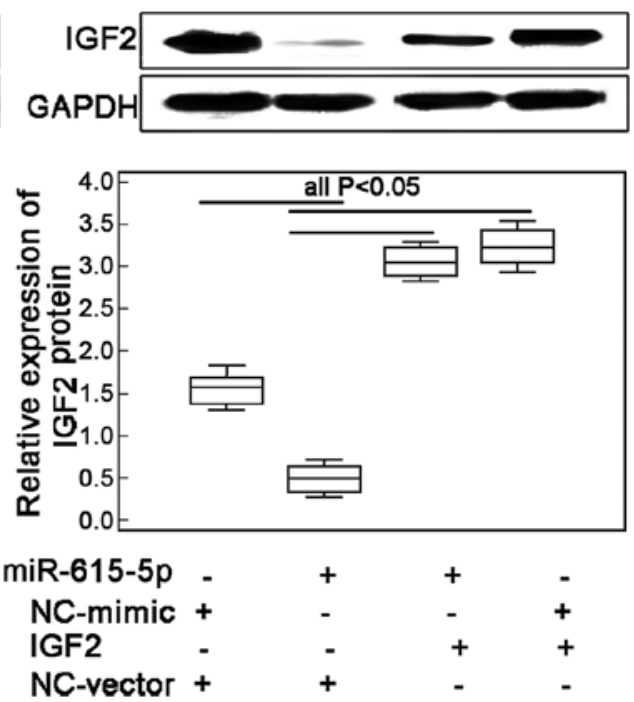

Figure 4. Relative expression levels of IGF2 protein in human ESCC cells co-transfected with miR-615-5p/NC-mimcs and IGF2/NC-expression vectors (A and B) Western blot analysis showed that the endogenous expression levels of IGF2 protein in ECA109 and KYSE410 cells transfected with the miR-615-5p mimic in the presence of IGF2 expression vector were both significantly higher than those co-transfected with the miR-615-5p mimic and vector control (both $\mathrm{P}<0.01$, respectively).

mRNA expression in ESCC tissues (Spearman's correlation: $r=-0.662, P<0.001$, Fig. 3E). These data offer evidence that IGF2 may be a direct target of miR-615-5p in human ESCC cells.

Restoration of IGF2 expression reverses the inhibitory effects of miR-615-5p in ESCC cell motility. To confirm whether miR-615-5p exerts its inhibitory effects on ESCC cell motility through regulating its target gene IGF2, the migrated and invasive capacities of ECA109 and KYSE410 cells with the co-transfection of miR-615-5p-mimics and Lv-CMV-GFP-IGF2 expression vector were further evaluated. The transfection efficiency was examined by western blot analysis. The results indicated that the endogenous expression levels of IGF2 protein in ECA109 and KYSE410 cells were both significantly increased following the transfection of the miR-615-5p mimic in the presence of IGF2 expression vector (both $\mathrm{P}<0.01$, Fig. $4 \mathrm{~A}$ and $\mathrm{B}$, respectively). Interestingly, the 

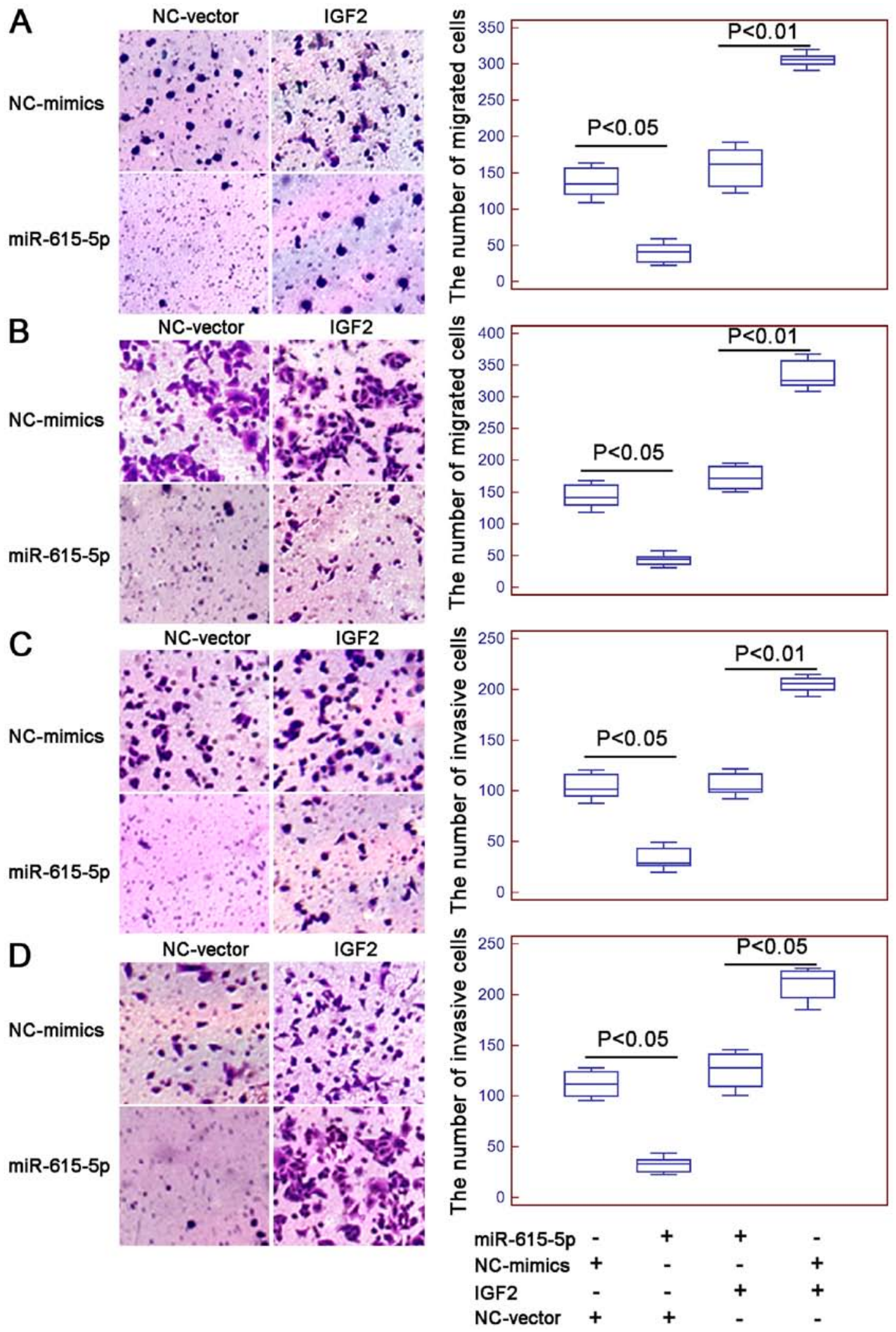

Figure 5. Restoration of IGF2 expression reverses the inhibitory effects of miR-615-5p in ESCC cell motility. (A and B) The number of migrated ECA109 and KYSE410 cells, respectively, co-transfected with miR-615-5p mimics and IGF2 expression vector was significantly larger than those with the co-transfection of miR-615-5p mimics and negative control expression vector (all $\mathrm{P}<0.05)$. (C and D) Transwell assays were performed to detect the functions of miR-615-5p in ESCC cell migration and invasion following the co-transfection with miR-615-5p mimics and IGF2 expression vector. The number of invaded ECA109 and KYSE410 cells, respectively, co-transfected with miR-615-5p mimics and IGF2 expression vector was significantly larger than those with the co-transfection of miR-615-5p mimics and negative control expression vector (all $\mathrm{P}<0.05$ ).

number of migrated and invaded ESCC cells with the overexpression of miR-615-5p and the restoration of IGF2 were both higher than those co-transfected with miR-615-5p mimics and vector control (all $\mathrm{P}<0.05$, Fig. 5). 


\section{Discussion}

miRNAs have recently become a new 'hot topic' in the research field of cancer-related molecular mechanisms. Increasing evidence indicates that miRNAs play crucial roles in carcinogenesis and cancer maintenance $(8,19)$. Since it is still a major challenge to identify molecular alterations in ESCC, we focused on this malignancy in the present study. Our data showed that the expression levels of miR-615-5p in ESCC tissues and cells were markedly higher than those in non-cancerous esophageal mucosa and human normal esophageal cells, respectively. Statistically, the downregulation of miR-615-5p was closely correlated with various aggressive clinicopathological features of patients with ESCC, such as advanced TNM stage, positive lymph node metastasis and moderate-poor differentiation. Functionally, we found that the re-expression of miR-615-5p suppressed the invasion and migration of ESCC cells. miRNAs exert the specific functions via inhibiting translation or promoting degradation of the corresponding target mRNAs using complementary sequences. Here, we identified IGF2 as a direct target gene of miR-615-5p, and also confirmed that the inhibitory effects of miR-615-5p in ESCC cell motility were reversed by the restoration of IGF2 expression. To the best of our knowledge, this is the first study to observe the expression pattern of miR-615-5p, its clinical significance and functional role in human ESCC.

miRNAs have been observed to be frequently expressed abnormally and function as 'onco(genic)-miRs' or 'tumorsuppressor miRs' in multiple malignant processes (20). These miRNAs may be good candidates as biomarkers for cancer diagnosis and prognosis (21). In most recent studies, miR-615-5p was identified as a tumor suppressor in various human cancers. For example, Sun et al (22) observed the downregulation of miR-615-5p in pancreatic ductal adenocarcinoma, and confirmed that low miR-615-5p expression was an independent prognostic marker for patients; Bai et al (23) found the decreased expression of miR-615 in breast cancer tissues and cell lines. In contrast, Wu et al (24) indicated that miR-615-5p was overexpressed in hepatocellular carcinoma tissues and cell lines; however, its overexpression could suppress both the cell proliferation and migration of hepatocellular carcinoma cells in vitro, suggesting that it is not necessarily the case that the overexpressed miRNAs act as oncogenes or promote tumorigenesis, and the downregulated miRNAs often play a tumor suppressive role. Consistent with these previous studies, our results here confirmed the reduced expression of miR-615-5p in both ESCC tissues and cell lines, and also indicated its contributions to cancer progression. To investigate whether the dysregulation of miR-615-5p is responsible for ESCC cell motility, the transfection of miR-615-5p mimics was performed in ESCC cell lines in vitro, and the Transwell assays showed that ESCC cell migratory and invasive potentials were suppressed by miR-615-5p, suggesting that the downregulation of miR-615-5p in ESCC cells may be a prerequisite for metastasis.

Since the miRNA actions are exerted through regulating the corresponding target genes, we also identified the candidate target genes of miR-615-5p in order to investigate the underlying mechanism of actions on how miR-615-5p abnormal expression impacts ESCC. IGF2, as an imprinted gene in mammals, has been observed to be overexpressed in multiple childhood and adult malignancies (25). Its upregulation is associated with resistance of chemotherapy and unfavorable prognosis in patients (26-28). Especially, Chava et al (29) performed immunohistochemistry to observe the expression patterns of IGF2 in 39 archival tissue samples of different esophageal pathologies, and found that IGF2 expression was enhanced in squamous cell carcinoma, adenocarcinoma, and dysplasia of squamous epithelium samples when compared with normal controls; Gao et al (30) discovered interaction between a long non-coding RNA (lncRNA) 91H and IGF2, and revealed that $91 \mathrm{H}$ contributed to the occurrence and progression of ESCC via suppressing the expression of IGF2. In the present study, our data demonstrated the IGF2 was directly regulated by miR-615-5p in ESCC. Using a luciferase reporter assay, we demonstrated that miR-615-5p directly targeted IGF2 by binding to its 3'UTR binding sites. Moreover, the inverse correlation between miR-615-5p and IGF2 mRNA expression in the ESCC tissues had statistical significance. Concomitantly, the inhibitory effects of miR-615-5p in ESCC cell migration and invasion were reversed by the enforced expression of IGF2, implying that miR-615-5p downregulation may be an important event related to IGF2 upregulation in human ESCC, and the reduced expression of miR-615-5p may promote cancer cell motility by influencing IGF2 expression.

In conclusion, our findings indicate miR-615-5p downregulation as an underlying molecular mechanism of development and progression, and as a potential therapeutic target of human ESCC. Also, we showed that the miR-615-5p/IGF2 axis may bring important contributions on cell motility of human ESCC.

\section{References}

1. Jemal A, Bray F, Center MM, Ferlay J, Ward E and Forman D: Global cancer statistics. CA Cancer J Clin 61: 69-90, 2011.

2. Enzinger PC and Mayer RJ: Esophageal cancer. N Engl J Med 349: 2241-2252, 2003.

3. Tan C, Qian X, Guan Z, Yang B, Ge Y, Wang F and Cai J: Potential biomarkers for esophageal cancer. Springerplus 5: 467, 2016.

4. Ohashi S, Miyamoto S, Kikuchi O, Goto T, Amanuma Y and Muto M: Recent advances from basic and clinical studies of esophageal squamous cell carcinoma. Gastroenterology 149: 1700-1715, 2015.

5. Kang X, Chen K,Li Y,Li J,D'Amico TA and Chen X: Personalized targeted therapy for esophageal squamous cell carcinoma. World J Gastroenterol 21: 7648-7658, 2015.

6. Makarova JA, Shkurnikov MU, Wicklein D, Lange T, Samatov TR, Turchinovich AA and Tonevitsky AG: Intracellular and extracellular microRNA: An update on localization and biological role. Prog Histochem Cytochem 51: 33-49, 2016.

7. Yan X, Xu H and Yan Z: Functional perspective and implications of gene expression by noncoding RNAs. Cancer Transl Med 1: $137-152,2015$.

8. Svoronos AA, Engelman DM and Slack FJ: OncomiR or tumor suppressor? The duplicity of microRNAs in cancer. Cancer Res 76: 3666-3670, 2016.

9. Rupaimoole R, Calin GA, Lopez-Berestein G and Sood AK: miRNA deregulation in cancer cells and the tumor microenvironment. Cancer Discov 6: 235-246, 2016.

10. Liu W, Li M, Chen X, Zhang D, Wei L, Zhang Z, Wang S, Meng L, Zhu S and Li B: MicroRNA-373 promotes migration and invasion in human esophageal squamous cell carcinoma by inhibiting TIMP3 expression. Am J Cancer Res 6: 1-14, 2015.

11. Nie J, Ge X, Geng Y, Cao H, Zhu W, Jiao Y, Wu J, Zhou J and Cao J: miR-34a inhibits the migration and invasion of esophageal squamous cell carcinoma by targeting Yin Yang-1. Oncol Rep 34: 311-317, 2015. 
12. Mao Y, Liu J, Zhang D and Li B: MiR-1290 promotes cancer progression by targeting nuclear factor I/X(NFIX) in esophagea squamous cell carcinoma (ESCC). Biomed Pharmacother 76: 82-93, 2015.

13. Jiang Y, Zhang Y, Li F, Du X and Zhang J: CDX2 inhibits pancreatic adenocarcinoma cell proliferation via promoting tumor suppressor miR-615-5p. Tumour Biol 37: 1041-1049, 2016.

14. Gao W, Gu Y, Li Z, Cai H, Peng Q, Tu M, Kondo Y, Shinjo K, Zhu Y, Zhang J, et al: miR-615-5p is epigenetically inactivated and functions as a tumor suppressor in pancreatic ductal adenocarcinoma. Oncogene 34: 1629-1640, 2015.

15. Song LJ, Zhang WJ, Chang ZW, Pan YF, Zong H, Fan QX and Wang LXPU: PU.1 is identified as a novel metastasis suppressor in hepatocellular carcinoma regulating the miR-615-5p/IGF2 axis. Asian Pac J Cancer Prev 16: 3667-3671, 2015.

16. Zhao ZG, Jin JY, Zhang AM, Zhang LP, Wang XX, Sun JG and Chen ZT: MicroRNA profile of tumorigenic cells during carcinogenesis of lung adenocarcinoma. J Cell Biochem 116: 458-466, 2015.

17. Chou CH, Chang NW, Shrestha S, Hsu SD, Lin YL, Lee WH, Yang CD, Hong HC, Wei TY, Tu SJ, et al: miRTarBase 2016: Updates to the experimentally validated miRNA-target interactions database. Nucleic Acids Res 44 (D1): D239-D247, 2016.

18. El Tayebi HM, Hosny KA, Esmat G, Breuhahn K and Abdelaziz AI: miR-615-5p is restrictedly expressed in cirrhotic and cancerous liver tissues and its overexpression alleviates the tumorigenic effects in hepatocellular carcinoma. FEBS Lett 586 : 3309-3316, 2012

19. Gulyaeva LF and Kushlinskiy NE: Regulatory mechanisms of microRNA expression. J Transl Med 14: 143, 2016.

20. Harada K, Baba Y, Ishimoto T, Shigaki H, Kosumi K, Yoshida N, Watanabe $\mathrm{M}$ and $\mathrm{Baba} \mathrm{H}$ : The role of microRNA in esophageal squamous cell carcinoma. J Gastroenterol 51: 520-530, 2016.
21. Arora A, Singh S, Bhatt AN, Pandey S, Sandhir R and Dwarakanath BS: Interplay between metabolism and oncogenic process: Role of microRNAs. Transl Oncogenomics 7: 11-27, 2015.

22. Sun Y, Zhang T, Wang C, Jin X, Jia C, Yu S and Chen J: MiRNA615-5p functions as a tumor suppressor in pancreatic ductal adenocarcinoma by targeting AKT2. PLoS One 10: e0119783, 2015.

23. Bai Y,Li J,Li J,Liu Y and Zhang B: MiR-615 inhibited cell proliferation and cell cycle of human breast cancer cells by suppressing of AKT2 expression. Int J Clin Exp Med 8: 3801-3808, 2015.

24. Wu X, Deng L, Tang D, Ying G, Yao X, Liu F and Liang G: miR-615-5p prevents proliferation and migration through negatively regulating serine hydromethyltransferase 2 (SHMT2) in hepatocellular carcinoma. Tumour Biol 37: 6813-6821, 2016.

25. Brouwer-Visser J and Huang GS: IGF2 signaling and regulation in cancer. Cytokine Growth Factor Rev 26: 371-377, 2015.

26. Bruchim I, Sarfstein R and Werner H: The IGF Hormonal Network in Endometrial Cancer: Functions, regulation, and targeting approaches. Front Endocrinol (Lausanne) 5: 76, 2014.

27. Chao W and D'Amore PA: IGF2: Epigenetic regulation and role in development and disease. Cytokine Growth Factor Rev 19: 111-120, 2008

28. Mu Q, Wang L, Yu F, Gao H, Lei T, Li P, Liu P, Zheng X, Hu X, Chen Y, et al: Imp2 regulates GBM progression by activating IGF2/PI3K/Akt pathway. Cancer Biol Ther 16: 623-633, 2015.

29. Chava S, Mohan V, Shetty PJ, Manolla Ml, Vaidya S, Khan IA, Waseem GL, Boddala P, Ahuja YR and Hasan Q: Immunohistochemical evaluation of p53, FHIT, and IGF2 gene expression in esophageal cancer. Dis Esophagus 25: 81-87, 2012.

30. Gao T, He B, Pan Y, Xu Y, Li R, Deng Q, Sun H and Wang S: Long non-coding RNA $91 \mathrm{H}$ contributes to the occurrence and progression of esophageal squamous cell carcinoma by inhibiting IGF2 expression. Mol Carcinog 54: 359-367, 2015. 